\title{
Dificultades en atención y memoria en alumnado de Educación Primaria con Trastorno por Déficit de Atención e Hiperactividad
}

\author{
Tania Vieites Lestón \\ Universidade da Coruña
}

\begin{abstract}
Resumen: Este estudio analiza las dificultades en atención y memoria de los escolares con Trastorno de Déficit de Atención e Hiperactividad (TDAH) entre los ocho y doce años (cursando cuarto, quinto y sexto de primaria), en comparación con un grupo control de la misma edad $(N=80)$, mediante la aplicación de tres pruebas estandarizadas: el test de percepción de diferencias o test de caras, el Children's Color Trails Test (CCTT) y la prueba de dígitos de la Escala de Inteligencia Wechsler. Estas pruebas analizan la atención selectiva, la atención sostenida, la atención alternante, la memoria a corto plazo y la memoria de trabajo, respectivamente. Los resultados muestran diferencias significativas entre el grupo control (grupo sin TDAH) y el grupo con TDAH en todos los procesos cognitivos evaluados. Además, se observan diferencias en las correlaciones de dichas funciones en ambos grupos, comprobando que puede existir una estrecha relación entre el déficit de atención y la ejecución de otros procesos cognitivos.
\end{abstract}

Palabras clave: Memoria, Atención, Procesos Cognitivos, TDAH, Educación Primaria.

Difficulties in attention and memory in Primary Education students with Attention Deficit Hyperactivity Disorder

\begin{abstract}
This investigation analyzes the difficulties in attention and memory of children with Attention Deficit Disorder and Hyperactivity (ADHD) between eight and twelve years old (attending fourth, fifth and sixth grade of primary school) compared with a control group of the same age $(n=80)$, by applying three standardized tests: the test of perception of differences or test face, Children's Color Trails Test (CCTT) and test digit by Wechsler Intelligence Scale. These tests analyze selective attention, sustained attention, alternating attention, short term memory and working memory, respectively. The results show significant differences between the control group (group without ADHD) and ADHD group in all evaluated cognitive processes. In addition, differences in the correlations of these functions in both groups, checking that there may be a close relationship between attention deficit and implementation of other cognitive processes.
\end{abstract}

Keywords: Memory, Attention, Cognitive Processes, ADHD, Primary Education.

El Trastorno por Déficit de Atención e Hiperactividad (TDAH), el cual se define como un trastorno del desarrollo neurológico que se caracteriza por síntomas de inatención, hiperactividad e impulsividad que son inconsistentes con el nivel de desarrollo y que repercuten de manera negativa en las

Recibido: 27/09/2018 - Aceptado: 11/02/2019 - Avance online: 19/03/2019

*Correspondencia: Tania Vieites.

Universidade da Coruña, Coruña, España

C.P: 15071, Coruña, España.

E-mail: t.vieites@udc.es

Vieites, T. (Avance Online).Dificultades en atención y memoria en alumnado de Educación Primaria con Trastorno por Déficit de Atención e Hiperactividad. Revista de Psicología y Educación, 14(2), 136-143, hitps://doi.org/10.23923/rpye2019.02.178 actividades sociales y académicas (DSM5, 2013). Esta sintomatología afecta en diferentes grados y con combinaciones distintas que, en muchos casos, se encuentran complementadas con trastornos comórbidos, tales como trastornos de conducta y/o dificultades de aprendizaje. "El TDAH se caracteriza por ser un trastorno heterogéneo" (Afonso, Artiles, Díaz, Jiménez y Rodríguez, 2013, p.155).

El TDAH es uno de los trastornos psiquiátricos infantiles más frecuentes (Macià, 
2012). Como indica Díaz et al. (2013) en su investigación sobre los estudios de prevalencia del trastorno, existen tasas de prevalencia muy variadas. Pero todos coinciden que en cuanto al género la tendencia es claramente favorable a un predominio de varones. La tasa de prevalencia del TDAH más citada entre los niños de edad escolar se encuentra en el $5 \%$ y el 2,5\% de los adultos (DSM-5).

Según la APA (2013), la ratio niños/niñas es de 4 a 1 en la población general y de 9 a 1 en la población clínica, diferencias que suelen desaparecer en la adolescencia. Los niños suelen mostrar unos síntomas más severos que las niñas, sobre todo la hiperactividad, mientras que en las niñas son los déficits atencionales (Barkley, 2006a; Biederman et al., 2004 citado en Lavigne y Romero, 2010). El DSM-5 (APA, 2013) ofrece tasas de prevalencia entre el 3 y el $7 \%$ en la población infantil entre 6 y 12 años pero la prevalencia más alta la encontramos en el rango de edad de 6-9 años. Por esta razón su estudio es fundamental para el desarrollo educativo de las personas afectadas.

Por otro lado, existen diferentes tipos de TDAH, es decir, que dicho trastorno engloba diferentes modalidades o tiene diferentes manifestaciones. El DSM-5 determina las tres siguientes; predominante inatento, predominante hiperactivo-impulsivo y presentación combinada. Todos los subtipos se reconocen bajo la etiqueta de trastorno por déficit de atención con hiperactividad (TDAH), aunque presente sólo déficit de atención o predomine el subtipo hiperactivo.

$\mathrm{Si}$ nos centramos en las diferentes presentaciones del TDAH, el subtipo más frecuente es el inatento aunque el combinado sea el más diagnosticado. En un estudio de metanálisis de Willcutt (2012) se pudo comprobar que la presentación inatenta es hasta dos veces más frecuente que la combinada en la edad escolar, aunque parezcan paradójicos los resultados es porque los afectados por la presentación combinada acuden antes a consulta, y por tanto se diagnostica con mayor frecuencia.

El TDAH entendido como un trastorno del desarrollo neurológico que afecta a nivel madurativo cerebral y a las áreas cognitivas del cerebro. Muestra que estas alteraciones en determinadas áreas del cerebro se traducen en procesos cognitivos disfuncionales que dan como resultado respuestas conductuales diferentes. Muchos de los síntomas y comportamientos de los estudiantes con TDAH son consecuencia de los problemas en los procesos cognitivos, perceptivos y neurobiológicos, siendo este abanico de comportamientos la expresión conductual y observable de un trastorno en el funcionamiento cognitivo (Artigas y Narbona, 2011). Los escolares con TDAH muestran mayor rigidez cognitiva en el procesamiento de la información, es decir, su flexibilidad cognitiva es menor. Mostrando déficit en la regulación de las emociones o dificultades para inhibir una respuesta.

Por un lado, los procesos atencionales son imprescindibles en las funciones ejecutivas de los escolares. Según destaca García (2014) estos procesos requieren de un mecanismo implicado directamente en la activación y el funcionamiento de los procesos $y / u$ operaciones de selección, distribución y mantenimiento de la actividad psicológica. Los niños con TDAH tienen un foco muy inconsciente y fácilmente cambiante (Guerrero, 2016).

$\mathrm{Se}$ determina que el funcionamiento atencional de los niños con TDAH es diferente al del resto de los niños. Pero no se trata de afirmar que los mecanismos atencionales del niño con TDAH no funcionen, sino más bien atienden de una manera diferente. Suelen tener dificultades en mantener la atención ante una tarea o estímulo, atención sostenida, sobre todo si son actividades poco motivadoras (Guerrero, 2016).

Por otro lado, los niños con TDAH no tienen dificultades en ningún tipo de atención involuntaria porque suelen prestar atención a todo tipo de estímulos. En cuanto a atención voluntaria se refiere cabe destacar que los niños con TDAH tienen dificultades para mantener la atención, ya que dicha atención depende de las funciones ejecutivas, esta dificultad depende de la motivación externa que reciba a la hora de realizar la actividad 
que le requiere dicha atención voluntaria. Barkley (2006) cree que la falta de atención que muestran los niños con TDAH inatento refleja las deficiencias en la rapidez del procesamiento de la información y en la atención selectiva o focalizada, mientras que los niños con TDAH con hiperactividad e impulsividad muestran dificultades en la atención sostenida y en su incapacidad para no atender a estímulos irrelevantes y los TDAH de tipo combinado tendrían déficits en la atención sostenida como en la dificultad de control motor (Macià, 2012).

Por otro lado, la memoria de trabajo (MT) es un sistema activo que mantiene y manipula la información, permitiendo que se lleven a cabo diversos procesos cognitivos como la lectura, el razonamiento o la comprensión lingüística (Portellano y García, 2014). En otras palabras, la MT se refiere a la capacidad de mantener y manipular por un corto periodo de tiempo la información necesaria para guiar una determinada conducta (Enseñat, 2015). Los escolares con TDAH poseen dificultades en la habilidad de retener en la mente aquella información necesaria para guiar las acciones, recordar hacer las cosas en un futuro cercano, dificultad para memorizar y seguir instrucciones, olvidar una información mientras trabaja en otra y, también, dificultad para manipular y transformar la información que almacena al servicio de guiar su conducta hacia un objetivo. Es decir, como afirma Díaz et al. (2013) los síntomas presentes en el TDAH ocasionan algún deterioro funcional en el alumnado que los presenta.

La investigación realizada en la Universidad Autónoma de Barcelona por García, Estévez y Junqué (2001), determina que los estudiantes con TDAH tienen dañadas sus habilidades amnésicas, es decir, tienen dificultades en la memoria de trabajo y en la memoria a corto plazo, en concreto, la memoria inmediata está en mayor medida más dañada.

Sin embargo, la memoria a corto plazo (MCP) se entiende como almacén de información, de capacidad limitada, que mantiene dicha información por un breve periodo de tiempo (Portellano y García,
2014). La MCP está relacionada directamente con la MT, ambas pueden verse afectadas en escolares con TDAH, ya que muestran deterioro en algunos procesos cognitivos. Cabe destacar que la MCP no se encuentra tan afectada como la MT, de acuerdo con Guerrero (2016) la memoria operativa es un tipo de MCP, diferenciándose de ésta en que la primera no sólo almacena información, como hace la $\mathrm{MCP}$, sino que también opera con ella para llegar a un resultado o meta.

La mayor parte de los estudios sobre TDAH se centran en el déficit de atención, la hiperactividad y la impulsividad de estos escolares. Por esta razón parece conveniente remarcar la importancia de realizar más estudios que analicen otras funciones ejecutivas como la MT, la MCP y la planificación. El objetivo de esta investigación es precisamente determinar las diferencias en cuanto a MT, MCP, atención sostenida, selectiva y alternante, en escolares de 8-12 años con TDAH.

Este estudio pretende responder a la siguiente pregunta: $\grave{L}$ Los escolares con TDAH de 8 a 12 años muestran mayor déficit en los diferentes tipos de atención y en la memoria de trabajo que los escolares sin TDAH de la misma edad? A partir de esta cuestión se establecen dos objetivos generales: estudiar la atención selectiva, sostenida y alternante, la memoria a corto plazo y la memoria de trabajo en niños con TDAH y comprobar si existen diferencias significativas entre los grupos.

\section{MÉTODO}

\section{PARTICIPANTES}

Los participantes son escolares de centros educativos de la Comunidad Autónoma de Madrid y de la provincia de A Coruña, todos ellos en España. La muestra fue elegida de forma intencionada según la disponibilidad de los centros, el diagnóstico de los escolares y el rango de edad del estudio. La muestra final estuvo compuesta por 80 escolares entre 8 y 12 años, 40 escolares con TDAH y 40 escolares sin TDAH, todos cursando cuarto, 
quinto y sexto de Educación Primaria. En el grupo con TDAH se afirma la prevalencia del sexo masculino con un total de 29 escolares niños $(72,5 \%)$ y 11 niñas $(27,5 \%)$ frente al grupo control que es homogéneo con un total de 20 escolares niños (50\%) y 20 escolares niñas (50\%). La edad media de la muestra es de 10,4 años en el grupo con TDAH y 10,3 años en el grupo sin TDAH.

\section{INSTRUMENTOS}

Test de Caras Revisado (Thurstone y Yela, 2012). El test de Caras es un test de percepción de diferencias que tiene como objetivo principal evaluar los aspectos perceptivos y atencionales en contextos de orientación y evaluación escolar, en concreto, la atención selectiva. Esta prueba consta de sesenta elementos gráficos, cada uno de ellos está formado por tres dibujos esquemáticos, dos de ellos son iguales; la tarea consiste en determinar cuál es diferente y tacharla. La interpretación de los resultados se realiza considerando el número de aciertos y el número de errores del alumno, estipulando como medida principal el rendimiento del estudiante el número de aciertos netos (aciertos menos errores). Esta versión revisada del test incluye baremos a partir de una muestra a nivel nacional que supera los doce mil escolares. El coeficiente de fiabilidad es un estadístico que indica la precisión o estabilidad de los resultados. Señala la cuantía en que las medidas de la prueba están libres de errores casuales. Así, un índice de fiabilidad de 0,90 quiere decir que, en la muestra y condiciones usadas, el 90 por 100 de la varianza de la prueba se debe a la auténtica medida y sólo el 10 por 100 a errores aleatorios.

Children's Color Trails Test (CCTT) (Llorente, Williams, Satz y D'Elia, 2003). CCTT es una prueba que consta de dos partes; parte A y parte B. La primera evalúa la atención sostenida mientras que la segunda evalúa la atención alternante. La parte $A$ se compone de los números del 1 al 25 que aparecen separados en dos colores, color rosa y color amarillo, de forma alterna y distribuida de una manera aleatoria. Mientras que la parte
B se compone de los números del 1 al 25 pero repetidos en ambos colores dónde el escolar tiene que unir de forma aleatoria los números por colores. En definitiva, la parte A es principalmente una prueba de atención visual que implican seguimiento perceptivo y una secuenciación simple, mientras que la parte B, debido a la secuencia alterna evalúa más directamente sistemas frontales de funcionamiento.

Prueba de Dígitos de la Escala de Inteligencia WISC-IV (Wechsler, 2005). La prueba de dígitos evalúa la memoria de trabajo, capacidad para retener temporalmente en la memoria cierta información, trabajar u operar con ella y generar un resultado. También se trata de una medida de la memoria a corto plazo y de la atención, evaluando la capacidad del escolar para retener diversos elementos que no tienen relación lógica entre sí. Debido a que la información auditiva debe recordarse y repetirse de manera oral en una secuencia adecuada. Esta prueba consta de dos tareas de aplicación independiente; "dígitos en orden directo" y "dígitos en orden inverso". La prueba de Dígitos presenta una adecuada consistencia interna, una buena fiabilidad test-retest y una adecuada validez de constructo.

En definitiva, el Test de Caras evalúa la atención selectiva, el Children's Color Trails Test evalúa la atención sostenida y alternante, mientras que la Prueba de Dígitos de la Escala de Inteligencia WISC-IV evalúa la memoria de trabajo y la memoria a corto plazo.

\section{PROCEDIMIENTO}

La recogida de datos fue realizada en su contexto educativo mediante la aplicación individualizada de los instrumentos seleccionados. La aplicación de los mismos se ha realizado por parte de una psicopedagoga externa a los centros educativos durante el horario lectivo. Tras una previa carta de presentación del estudio a las familias de los escolares/as y su correspondiente autorización se realiza la evaluación de los procesos cognitivos de manera individual, fuera del 
aula, en el horario indicado por los tutores del alumnado. La selección de los centros se ha realizado mediante muestreo intencional según la disponibilidad y actitud positiva a participar en el estudio, por otro lado, la selección de la muestra ha sido realizada por los centros educativos según la autorización afirmativa de las familias a participar en el estudio.

\section{ANÁLISIS DE DATOS}

Se emplearon técnicas descriptivas para el análisis global de resultados, además de realizar una comparación de medias de las variables principales a analizar en este estudio (memoria de trabajo, memoria a corto plazo, atención selectiva, atención sostenida y atención alternante) mediante la prueba $t$ de Student y el estudio de la correlación entre diferentes variables mediante el coeficiente de correlación $r$ de Pearson. Los análisis fueron realizados a través del programa IBM Statistics 19.0 En todos los casos se ha tomado el valor 0.05 como valor de significación que corresponde con un nivel de confianza del 95\%.

\section{RESULTADOS}

En primer lugar, se realizó un análisis descriptivo de las puntuaciones medias globales de los participantes en las diferentes pruebas estandarizadas empleadas. La información relativa a este análisis puede verse en la Tabla 1.

Como se observa, el índice de impulsividad del Test de caras, que mide la atención selectiva de esta muestra, la diferencia de medias es significativa, $(p<0,005)$. En cuanto a la atención sostenida y la atención alternante del grupo control, medida en segundos mediante la prueba CCTT, dónde la media de la prueba CCTT 2 es mayor que la parte CCTT 1, con una diferencia significativa, $(p<0,005)$. Esto se debe a que la segunda parte tiene una dificultad mayor. En el grupo con TDAH se puede apreciar valores superiores en esta variable, demostrando que al igual que el grupo control, los escolares con TDAH alcanzan puntuaciones menores en atención alternante que en atención sostenida, dónde la media de la prueba CCTT 2 es mayor que la parte CCTT 1.

Tabla 1

Estadísticos descriptivos por grupos

\begin{tabular}{|c|c|c|c|c|c|c|c|}
\hline & Grupo & $\mathrm{n}$ & Media & $\begin{array}{c}\text { Desviación } \\
\text { típica }\end{array}$ & $\begin{array}{c}\text { t de } \\
\text { Student }\end{array}$ & $p$ & $d$ \\
\hline \multirow[t]{2}{*}{$\begin{array}{l}\text { Test caras (atención } \\
\text { selectiva) }\end{array}$} & Control & 40 & 13.66 & 26.83 & -2.45 & .01 & .56 \\
\hline & TDAH & 40 & -4.54 & 37.97 & & & \\
\hline \multirow[t]{2}{*}{$\begin{array}{l}\text { CCTT } 1 \text { segundos (atención } \\
\text { sostenida) }\end{array}$} & Control & 40 & 60.90 & 17.95 & 2.72 & .01 & .62 \\
\hline & TDAH & 40 & 82.15 & 45.98 & & & \\
\hline \multirow{2}{*}{$\begin{array}{l}\text { CCTT } 2 \text { segundos (atención } \\
\text { alternante) }\end{array}$} & Control & 40 & 118.12 & 28.08 & 3.38 & .01 & .77 \\
\hline & TDAH & 40 & 150.20 & 52.94 & & & \\
\hline \multirow[t]{2}{*}{$\begin{array}{l}\text { Dígitos orden directo } \\
\text { (memoria a corto plazo) }\end{array}$} & Control & 40 & 8.90 & 1.98 & -2.11 & .04 & .48 \\
\hline & TDAH & 40 & 8.05 & 1.57 & & & \\
\hline \multirow[t]{2}{*}{$\begin{array}{l}\text { Dígitos orden inverso } \\
\text { (memoria de trabajo) }\end{array}$} & Control & 40 & 6.92 & 1.46 & -2.27 & .03 & .51 \\
\hline & TDAH & 40 & 6.10 & 1.78 & & & \\
\hline
\end{tabular}


Por otro lado, la prueba de dígitos alcanza puntuaciones más altas en orden directo (memoria a corto plazo) que en orden inverso (memoria de trabajo) según la media de ambas pruebas en los dos grupos. Por lo tanto, los alumnos de la muestra tienen resultados inferiores en memoria de trabajo que en memoria a corto plazo, y la atención alternante tiene unos resultados inferiores que la atención sostenida en el grupo sin TDAH. Comprobando que existen diferencias significativas entre los grupos, con un nivel de significación $(p<.05)$.

En resumen, en todas las pruebas, el grupo sin TDAH (grupo control) supera la media con respecto al grupo con TDAH. Cabe destacar que la prueba CCTT está en segundos, por eso la media del grupo con TDAH es mayor, porque tardan más tiempo en realizar la actividad.

Además de la diferencia de medias, se observan diferencias significativas entre los diferentes procesos cognitivos a partir del valor de $p$, mediante la prueba $t$ para muestras independientes. Las resultados de todas las pruebas estandarizadas empleadas muestran un nivel de significación $(p<.05)$ indicando que existen diferencias significativas entre los grupos en todos los procesos cognitivos evaluados (atención selectiva, sostenida y alternante, memoria de trabajo y memoria a corto plazo).

Por otro lado, se ha realizado un análisis mediante el coeficiente de correlación de Pearson, el cual permite cuantificar el grado de relación existente entre variables del estudio en función de los procesos cognitivos evaluados. En el grupo control, se observan correlaciones entre los índices de las pruebas que evalúan atención sostenida y atención selectiva. Mientras que en el grupo con TDAH, la memoria a corto plazo tiene una correlación significativa y negativa con la atención sostenida $\left(r^{2}=-.37^{*}\right)$ $(p<.05)$. Además entre la memoria de trabajo y la memoria a corto plazo, ambas evaluada por la prueba de dígitos, existe una correlación negativa $\left(r^{2}=-.34^{*}\right)(p<.05)$.

\section{DISCUSIÓN}

Los resultados generales observados en este estudio permiten conocer los déficits característicos del TDAH y las diferencias que pueden existir entre los escolares diagnosticados con TDAH y los escolares sin dicho trastorno en los ejes principales de esta investigación.

Los objetivos del estudio eran estudiar tres tipos de atención (atención selectiva, sostenida y alternante), la memoria de trabajo y la memoria a corto plazo de escolares con TDAH además de analizar las diferencias y similitudes entre este grupo y el grupo control, sin TDAH. El presente estudio amplía los hallazgos obtenidos en investigaciones precedentes sobre el TDAH.

En primer lugar, los niños con TDAH muestran un rendimiento menor que los escolares del grupo control en el tiempo de reacción, respondiendo más lentamente, resultados obtenidos a través de la prueba CCTT que evalúa atención sostenida y atención alternante. Estos resultados muestran que los escolares con TDAH tienen mayor déficit atencional, tal y como indica la bibliografía actual y los criterios diagnósticos de dicho trastorno. Además, en la evaluación de la atención sostenida se han encontrado diferencias notables entre ambos grupos que, según Barkley (1997), se relacionan claramente con una disfunción atencional.

En segundo lugar, los resultados permiten concluir que el rendimiento de los niños con TDAH en la prueba de dígitos, sobre todo en dígitos en orden inverso, es inferior que el de los escolares del grupo control. Estos datos son coherentes con la literatura existente (Hale et al., 2002), esta prueba mide la memoria de trabajo y memoria a corto plazo como en este estudio y en otras investigaciones (García et al., 2001), estudios cuyos frutos han puesto de manifiesto unos resultados inferiores de los escolares con TDAH frente a los de los niños que no padecen dicho trastorno. Estos resultados en los escolares con TDAH en la prueba de dígitos se pueden interpretar como un reflejo de los problemas de orientación y focalización atencional de la memoria operativa, componente que controla y supervisa los sistemas subsidiarios verbal y viso-espacial, estando el primero de ellos implicado en la ejecución de la prueba 
de dígitos en orden inverso (Baddeley 1999, citado en Balluerka et al., 2009).

En tercer lugar, Narbona y CrespoEguilaz (2005) destacan que la atención sostenida y la memoria de trabajo actúan de forma sincronizada para dar continuidad y congruencia a la actividad mental y a la conducta humana. En nuestro estudio, la asociación entre la atención sostenida y la memoria de trabajo en el grupo con TDAH es muy elevada, corroborando dicha afirmación.

Por otro lado, cabe destacar que los participantes del estudio con TDAH muestran características similares, en lo referente a promocionar curso y a la medicación de los escolares. Es decir, la mayor parte de los escolares combinan la intervención educativa en el aula con el tratamiento farmacológico, repercutiendo de forma favorable en sus estudios. Esto se comprueba mediante la comparación de medias para muestras independientes, donde se observa que los escolares medicados diariamente muestran menor déficit en los procesos cognitivos evaluados, excepto una pequeña diferencia en la prueba que evalúa memoria de trabajo. Además, un $32,5 \%$ de los escolares con TDAH no promocionan curso, repitiendo curso al menos una vez, esto nos indica que si la intervención psicoeducativa no resulta satisfactoria o no se alcanzan los contenidos mínimos del curso, el escolar repite curso.

Por tanto, a partir de los resultados obtenidos se puede concluir que los niños con TDAH tienen un rendimiento menor que los niños del grupo control en las pruebas que evalúan atención, y en menor medida, muestran déficits en los procesos cognitivos evaluados mediante la prueba de dígitos. Mostrando que para los escolares con TDAH es más difícil focalizar y mantener la atención que llevar a cabo tareas que requieran la implicación de la memoria de trabajo. Alsina y Sáiz (2003) señalan que cuanto menor es la velocidad de procesamiento, menor es la duración de la información en la memoria de trabajo, proceso que con la atención es esencial para lograr concentración en las operaciones cognitivas y actividades intencionales.
En resumen, este estudio responde de forma positiva al problema a investigar, mostrando diferencias entre ambos grupos, al igual que otras investigaciones de la misma temática. Se puede afirmar el déficit atencional característico del trastorno, además de la asociación de este déficit con otros procesos cognitivos como los evaluados en este estudio, la memoria de trabajo y la memoria a corto plazo en los escolares de 8 a 12 años.

A partir de estos resultados, desde el ámbito de la orientación educativa, se debe fomentar una intervención cognitiva y psicoeducativa que intente entrenar y mejorar los procesos cognitivos de los niños con TDAH, promoviendo una intervención integral. Para esto es necesario intervenir, de forma colaborativa, con los padres, el niño y la escuela para alcanzar unos resultados terapéuticos favorables. Las intervenciones psicoeducativas a los padres y al entorno social-educativo del niño disminuyen los problemas de conducta del niño y favorecen el manejo de los síntomas del TDAH. Además, la intervención escolar es esencial en los niños con TDAH. Como corrobora el estudio de Areces, Cueli, García, Peláez y Rodríguez (2017), una intervención adecuada fomenta el aprendizaje de estrategias que favorecen las habilidades atencionales.

Para concluir, se considera necesario destacar la importancia que tiene seguir investigando sobre el trastorno con el fin de mejorar el diagnóstico y el tratamiento de los niños que presentan TDAH. La profesionalidad y el interés por el bienestar y la salud de la persona son los que deben dirigir el rumbo futuro de la investigación sobre este trastorno. Además de solventar las limitaciones de este estudio, como por ejemplo; ampliar la muestra a estudiar, tener en cuenta el subtipo de TDAH (combinado, inatento e hiperactivoimpulsivo) o el hecho de que los grupos no sean equivalentes en cuanto a género.

\section{- Conflicto de intereses.}

Los autores declaran no tener ningún conflicto de intereses. 


\section{REFERENCIAS}

Alsina, Á. Y Sáiz, D. (2003): Un análisis comparativo del papel del bucle fonológico versus la agenda viso-espacial en el cálculo en niños de 7-8 años, Psichotema, 15 (2), 241-246.

American Psychiatric Association., Kupfer, D.J., Regier, D.A., Arango López, C., AyusoMateros,J.L., Vieta Pascual, E., \& Bagney Lifante, A. (2013). DSM-5: Manual de Diagnóstico y estadístico de los trastornos mentales ( $5^{a}$ ed). Madrid: Editorial Médica Panamericana.

Artigas, J. y Narbona, J. (201 1). Trastornos del neurodesarrollo. Barcelona: Viguera.

Balluerka, N., Iraola, J.A., Soroa, G. y Soroa, M. (2009). Evaluación de la atención sostenida de niños con Trastorno de Déficit de Atención. Revista de Psicodidáctica, 14 (2), 279-297.

Barkley, R.A. (1997). Behavioral inhibition, sustained attention and executive functions: Constructing a unifying theory of ADHD. Psychological Bulletin, 121, 65-94.

Barkley, R.A. (2006). Attention-deficithyperactivity disorder: a handbook for diagnosis and treatment. New York: Guilford Press.

Díaz, A., Jiménez, J.E., Rodríguez, C., Afonso, M. y Artiles, C. (2013). Consideraciones de los estudios de prevalencia del trastorno por déficit de atención con o sin hiperactividad (TDAH). Revista de Psicología y Educación, 8 (2), 155-170.

Enseñat, A. (2015). Neuropsicología pediátrica. Madrid: Síntesis.

García, C., Estévez, A. y Junqué, C. (2001). Perfil de memoria en el trastorno por déficit de atención con hiperactividad. Anuario de Psicología, 32(4), 35-46.

García, J. (2014). Psicología de la atención. Madrid: Síntesis.
Guerrero, R. (2016). Trastorno por déficit de atención con hiperactividad. Entre la patología y la normalidad. Barcelona: Planeta.

Hale, J.B., Hoeppner, J.B. \& Fiorello, C.A. (2002). Analyzing digit span components for assessment of attention processes. Journal of Psychoeducational Assessment, 20,128-143.

IBM Corp.Rekeased (2010). IBM SPSS Statistic for Windows, Version 19.0. Armork, NY: IBM Corp.

Lavigne,R. y Romero, J.F. (2010). El TDAH. Madrid: Pirámide.

Llorente, A. M., Williams, J., Satz, P. \& D'Elia, L. F. (2003). Children's Color Trails Test professional manual. Lutz, FL: Psychological Assessment resources.

Macià, D. (2012). TDAH en la infancia y la adolescencia. Madrid: Pirámide.

Narbona, J. y Crespo-Eguilaz, N. (2005). Trastornos de memoria y de atención en disfunciones cerebrales del niño. Revista de Neurología, 40, 33-36.

Peláez, N., Cueli, M., Areces, D., García, T. y Rodríguez, C. (2017). Efecto de la representación dinámica integrada sobre la competencia matemática y la atención en niños con TDAH. Revista de Psicología y Educación, 12 (2), 105-115.

Portellano, J.A. y García, J. (2014). Neuropsicología de la atención, las funciones ejecutivas y la memoria. Madrid: Síntesis.

Thurstone, L.L. y Yela, M. (2012). Test de Percepción de Diferencias Revisado (CARAS-R). Madrid: TEA.

Wechsler, D. (2005). Escala de Inteligencia Wechsler para Niños IV. (WISC-IV). Madrid: TEA.

Willcut, E.G. (2012). The Prevalence of DSMIV Attention-Deficit/Hyperactivity Disorder: A Meta-Analytic Review. Neurotherapeutics, 9, 490-499. 